\title{
Innovative Acoustic Treatments of Nacelle Intakes Based on Optimised Metamaterials
}

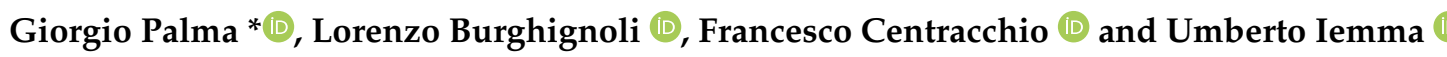 \\ Department of Engineering, University of Roma Tre, Via Vito Volterra, 62, 00146 Roma, Italy; \\ lorenzo.burghignoli@uniroma3.it (L.B.); francesco.centracchio@uniroma3.it (F.C.); \\ umberto.iemma@uniroma3.it (U.I.) \\ * Correspondence: giorgio.palma@uniroma3.it
}

Citation: Palma, G.; Burghignoli, L.; Centracchio, F.; Iemma, U. Innovative Acoustic Treatments of Nacelle Intakes Based on Optimised Metamaterials. Aerospace 2021, 8, 296. https://doi.org/10.3390/ aerospace 8100296

Academic Editor: Lawrence S. Ukeiley

Received: 15 July 2021

Accepted: 11 October 2021

Published: 14 October 2021

Publisher's Note: MDPI stays neutral with regard to jurisdictional claims in published maps and institutional affiliations.

Copyright: (C) 2021 by the authors. Licensee MDPI, Basel, Switzerland. This article is an open access article distributed under the terms and conditions of the Creative Commons Attribution (CC BY) license (https:// creativecommons.org/licenses/by/ $4.0 /)$.

\begin{abstract}
Modern turbofans with high bypass ratios, low blade passage frequencies and short nacelles require continuous development of acoustic linings to achieve the noise reductions expected by the international aviation authorities. Metamaterials and metafluids have been recently proposed as promising technologies for designing innovative acoustic treatments dedicated to reducing aeronautic turbofan noise emissions. In this work, a phase-gradient metasurface treatment is investigated as a way to tackle the noise radiation from an axially symmetric nacelle. This paper aims to study the potential benefits of the mentioned technology, and is not an attempt to design a complete new liner or nacelle. The metasurface is modelled through an equivalent metafluid, and a simulation-based optimisation is used in defining the design parameters. The tonal contribution of the blade passage frequency is considered, and the numerical results with the metafluid optimised on one azimuthal mode at a time show a significant effect in terms of acoustic levels and directivity over an arc of virtual receivers.
\end{abstract}

Keywords: metamaterials; metasurfaces; metafluids; optimisation; simulation-based design; acoustics; liners; engine noise

\section{Introduction}

Massive efforts have been devoted to alleviating noise pollution produced by aviation during the last decades by the aeroacoustic community. Urban areas surrounding airports are continuously expanding, and air traffic, even though momentary slowed by the Covid19 pandemic, is forecast to increase by all future-predicting scenarios. Both these issues have to be tackled for the sustainable development of the air transport system.

The research on community noise reduction has been strongly driven by the ACARE goals of Vision 2020 and Flightpath 2050 [1], which aim at reducing the perceived noise of new aircraft dramatically with an abatement of $65 \%$ compared to the 2000 baseline configuration. One of the possible approaches to the issue of noise reduction is eliminating the main source or at least substituting it with a quieter one. Since their introduction in the early 1970s, turbofans contributed dramatically to increasing engine efficiency and reducing noise emissions. The increasing importance of the fan in thrust generation changed the distribution of the relative noise level emissions between the various sources of the engine. As higher bypass ratios led to decreasing importance of the jet component, the fan noise emerged as the new dominant source, particularly during take-off operations [2].

Huge research has been conducted on low noise technologies for engine components to alleviate the aviation-related community noise, e.g., the acoustic liners that extensively cover the inner surfaces of modern turbofan nacelles, being the single-degree-of-freedom liners particularly effective for tonal noise abatement [3], acting on one of the primary sources on aircraft. The evolution of turbofans, however, is leading towards a further increase of the bypass ratio and fan diameters, as confirmed by the comparison between CFM International Leap and CFM-56 or the General Electric GE9x with GE90. High 
Bypass Ratio designs dominate modern commercial aviation, and the future generation of turbofans currently being developed, named Ultra High Bypass Ratio, are expected to have BPR up to 15 or higher. As a consequence of larger fan diameters, the tonal components from the fan are shifted down in the noise emission spectrum, with lower blade passage frequencies (BPFs). In addition, to partially compensate for the drag increase due to bigger sections, the future large engines are expected to have lower lined-length-to-diameter ratios, adding difficulties to reducing noise at the source.

New, yet standard, liners, e.g., with folded channels or multi-degree of freedom, are currently under development to tackle the aforementioned issues [4,5], and highly innovative concepts such as metamaterial-based acoustic treatments are being imagined [6,7]. The potential of metamaterials in aeroacoustics has been recently indicated [8,9] and shown in several works [10-16]. The particular metamaterials class of the phase-gradient metasurfaces (PGMSs), devoted to sound redistribution more than absorption, has been used to significantly change the noise emission directivity of simple $2 \mathrm{D}$ rectangular ducts in numerical simulations [17,18], and, while some technological challenges still remain open, possible synergies with standard liners were speculated. An analytic equivalence between a PGMS and a metafluid designed within the Transformation Acoustics framework was developed in $[18,19]$. The metafluid model demonstrated to be a computationally efficient method to address the simulation-based optimisation of the metasurface parameters, enlightening also new paths for building PGMS devices still unexplored. The PGMS-metafluid model is extended in this article, considering 3D axial symmetric devices with curvatures in the axial direction. The update, derived in Section 2, is necessary when dealing with geometries representing realistic nacelles of aeronautic turbofans and their inlet section as it is done in this work. The equivalent metafluid is, in fact, used to estimate, through numerical simulations, the effect of a PGMS acoustic lining on the noise radiated from a reference turbofan inlet, whose geometry is comparable with the modern high-bypass ratio aeronautical engines.

The interest in this paper is focused on the effects achievable by a phase-gradientbased treatment on the directivity of a nacelle-like duct. The topic is relatively new and fundamental research is still ongoing; this work, hence, is not dealing with the detailed design of a liner and the complete assessment of its performances. Three cases are analysed using as noise sources interacting with the metamaterial treatment three acoustic modes with different characteristics chosen among the propagating ones at the fan section for the BPF. For each case, the metafluid is designed through a single objective simulation-based optimisation, minimising the acoustic levels over an arc of virtual receivers surrounding the nacelle inlet for the single selected azimuthal mode.

Results from simulations in a quiescent fluid, comparing the lined and the reference configurations, are presented in Section 4, along with comments in Section 5, confirming the potential of this class of metamaterial linings.

\section{Theoretical Modelling of PGMS}

At the beginning of their development in acoustics, PGMS have been studied to overcome the Snell's law and the law of reflection, introducing an additional term in the evaluation of the reflection/refraction angle depending on the metasurface design, as described by the Generalised Snell's law (GSL)

$$
n_{t} \sin \theta_{t}=\frac{\lambda}{2 \pi} \frac{\partial}{\partial x} \Delta \phi(x, \lambda)+n_{i} \sin \theta_{i}
$$

Without losing generality, the equation is stated for a 1D phase delay gradient, in which $\lambda$ is the wavelength of the incident acoustic perturbation, $\theta_{i}$ and $\theta_{t}$ are the angles of the incident and transmitted fields, $n_{i}$ and $n_{t}$ are the refractive indexes of the incident and transmitted domains. In the case of a sound-hard boundary, where the only reflection can occur, Equation (1) reads

$$
\sin \theta_{r}=\frac{\lambda}{2 \pi} \frac{\partial}{\partial x} \Delta \phi(x, \lambda)+\sin \theta_{i}
$$


where $\theta_{r}$ is the reflection angle of the acoustic wave impinging on the boundary (see Figure 1a).

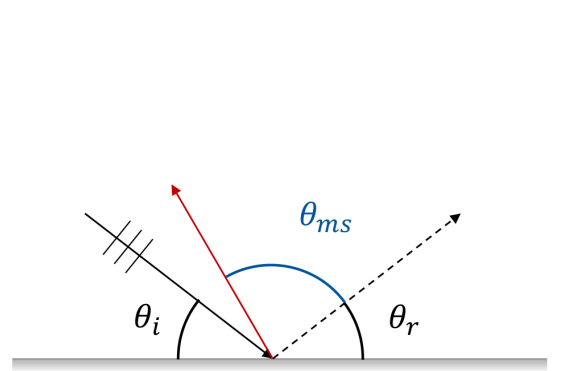

(a)

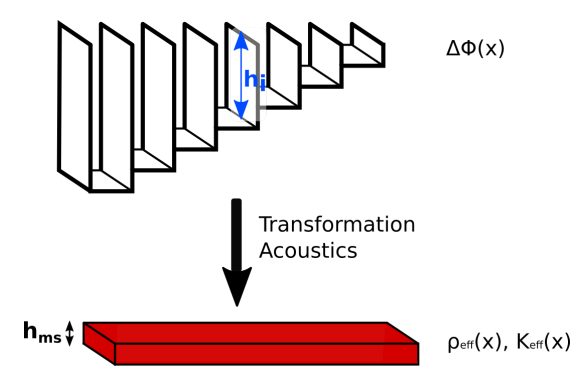

(b)

Figure 1. From phase gradient to effective metamaterial parameters using TA. The phase delay distribution determines the reflection angle from the boundary for a given incidence (a). The phase delay given by a cell is modelled with equivalent density and bulk modulus (b).

In both Equations (1) and (2), $\Delta \phi(x, \lambda)$ refers to the difference between the phase of the transmitted/reflected field from a metasurface-lined boundary and a standard one modelled by a perfectly sound-reflecting boundary $\Delta \phi=\phi_{m s}-\phi_{h w}$. In the following, only the case of reflection from a PGMS boundary will be considered. The typical implementation of a PGMS uses periodic arrangements of elementary cells capable of introducing a local $\Delta \phi$ in the reflected field. The phase gradient profile required for the application is reproduced with a spatial definition defined by the cells' dimensions by distributing the unit cells. Many concepts for the elementary cell and its design procedure have been proposed [20-26]; some of these designs have complex shapes, with several parameters controlling the geometries. However, they all have been conceived using the concept of the acoustic waveguide. In fact, the easiest way to achieve a phase delay gradient in the reflected field with respect to a flat hard wall is substituting it with a sequence of rectilinear waveguides for the zeroth-order mode with tailored lengths (see Figure 1).

The length of the tube $h$ can be easily linked to the phase delay achieved by a simple relation involving the wavelength $\lambda$ of the acoustic perturbation under consideration and the length of the extra path covered by the impinging wave, $2 h$ :

$$
\Delta \phi(x, \lambda)=-\frac{4 \pi h(x)}{\lambda} .
$$

A metafluid can achieve the same effect through the definition of its parameters, bulk modulus and inertia tensor. The Transformation Acoustic (TA) framework can be used to establish a design procedure for a metafluid that mimics a PGMS.

Following the TA procedure [27], the equation invariance under coordinate transformation is exploited to obtain the metafluid general governing equation for acoustic perturbations.

Let $\Omega$ and $\omega$ be the original and the deformed (metafluid) domains, the coordinates in each configuration are $\mathbf{X}$ and $\mathbf{x}$, respectively; the mapping $\Omega \rightarrow \omega$ is defined by $\mathbf{x}=\chi(\mathbf{X})$ and it is a one-to-one invertible transformation. The deformation gradient is $F=\bar{\nabla} \mathbf{x}$, or in component form $F_{i j}=\partial x_{i} / \partial X_{j}$; hence, the Jacobian of the deformation is $\Lambda=\operatorname{det} \mathbf{F}$. The polar decomposition implies $\mathbf{F}=\mathbf{V R}$ where $R$ is proper orthogonal and $\mathbf{V}^{\mathbf{2}}=\mathbf{F F}^{\mathbf{t}}$. Being $\mathbf{Q}$ a symmetric and divergence-free second-order tensor, the expression for the Laplacian in $\mathbf{X}$ in terms of derivatives in $\mathbf{x}$ is

$$
\bar{\nabla}^{2} f=\Lambda \mathbf{Q}: \nabla \Lambda^{-1} \mathbf{Q}^{-1} \mathbf{V}^{2} \nabla f .
$$

Hence, the scalar wave equation governing the acoustic field in $\Omega$, occupied by a homogeneous fluid with density $\rho_{0}$ and bulk modulus $\kappa_{0}$, 


$$
\frac{\partial^{2} p}{\partial t^{2}}-\frac{\kappa_{0}}{\rho_{0}} \bar{\nabla}^{2} p=0, \quad \mathbf{x} \in \Omega
$$

is, in the deformed region $\omega$ occupied by a metafluid, replicated by

$$
\frac{\partial^{2} p}{\partial t^{2}}-\kappa \mathbf{Q}: \varrho^{-1} \mathbf{Q} \nabla p=0, \quad \mathbf{x} \in \omega
$$

being $\kappa$ the metafluid bulk modulus and $\varrho$ its inertia tensor.

Axial Symmetric Geometries with Axial Curvature-PGMS-Metafluid in Nacelles

Without loss of generality, in the following, the problem is addressed under the hypothesis of inertial metafluid, i.e., $\mathbf{Q}=\mathbf{I}$ [27], a special case of the more general class of metacontinua. In this case, the bulk modulus and the inertia tensor are $\kappa=\kappa_{0} \Lambda$, $\varrho=\rho_{0} \Lambda \mathbf{V}^{-2}$. In this section, we analyse the case of an axial symmetric geometry, in which one of the boundaries defining the revolution section includes a PGMS lining. A coordinate transformation is set for each $i$-th tube/cell, following Figure 1b, to obtain a metafluid equivalent to a PGMS.

Considering two sets of cylindrical coordinates, $\mathbf{x}=(r, \alpha, z)$ and $\mathbf{X}=(R, A, Z)$ in the undeformed and deformed domain, respectively, the transformation reads

$$
\begin{aligned}
& R-r_{0}(z)=\frac{h_{m s}}{h_{i}}\left(r-r_{0}(z)\right) \quad r_{0}(z) \leq r \leq r_{0}(z)+h_{i} \\
& A=\alpha \\
& Z=z
\end{aligned}
$$

indicating with $r_{0}(z)$ the inner radius of the lined wall, which is, in general, a function of $z$, with $h_{i}$ the height of the $i$-th undeformed tube and with $h_{m s}$ the height of the metafluid lining, i.e., of the deformed channels. In this case, the deformation gradient $\mathbf{F}$ is

$$
\begin{aligned}
\mathbf{F} & =\left[\begin{array}{ccc}
f_{11} & 0 & f_{13} \\
0 & f_{22} & 0 \\
0 & 0 & f_{33}
\end{array}\right] \\
f_{11} & =\frac{h_{m s}}{h_{i}} \\
f_{13} & =\left(1-\frac{h_{m s}}{h_{i}}\right) \frac{\partial}{\partial z} r_{0}(z) \\
f_{22} & =\frac{h_{m s}}{h_{i}} \frac{R}{R-r_{0}(z)+\frac{h_{m s}}{h_{i}} r_{0}(z)} \\
f_{33} & =1
\end{aligned}
$$

hence, the bulk modulus and the inertia tensor are

$$
\begin{aligned}
\kappa_{i} & =f_{11} f_{22} \kappa_{0} \\
\boldsymbol{\varrho}_{i} & =f_{11} f_{22}\left[\begin{array}{ccc}
\frac{1}{f_{11}^{2}} & 0 & -\frac{f_{13}}{f_{11}^{2}} \\
0 & \frac{1}{f_{22}^{2}} & 0 \\
-\frac{f_{13}}{f_{11}^{2}} & 0 & \frac{f_{13}}{f_{33}}+1
\end{array}\right] \rho_{0} .
\end{aligned}
$$

It is worth stressing that, with this particular transformation, the anisotropy might even be transferred entirely to $\mathbf{Q}=\Lambda^{-1} \mathbf{V}$, obtaining a pentamode material with isotropic inertia [27]. 
From Equations (3) and (9), a link between GSL and metafluid theory is obtained as the phase delay of a cell is a function of the bulk modulus of the metafluid for a given transformation:

$$
\Delta \phi_{i}(\lambda)=\frac{4 \pi g_{1}\left(\kappa_{i}\right)}{\lambda}, \quad k_{i}=g_{2}\left(R, h_{m s}, h_{i}, r_{0}(z)\right)
$$

The above relation ensures that the effects predicted by the GSL are attainable by (ST)TA designed metamaterials. It also opens new ways to practically realise PGMS: all the concepts able to change the effective density and speed of sound of a domain, e.g., pentamode materials which already have been demonstrated to be effective in realising TA concepts [28,29], can be adopted for the design of a PGMS. Equation (10) is also beneficial from the numerical point of view when dealing with simulation-based optimisation problems. By parametrising the cells' responses through effective fluid parameters, there is no need for changing their geometry and regenerating the corresponding mesh at each iteration.

\section{Numerical Model}

As anticipated above, the studied configuration is a $2 \mathrm{D}$ axial symmetric geometry representative of a modern turbofan inlet portion. Following Figure 2, the reference engine fan radius is set $r_{n}=1.7 \mathrm{~m}$; the sizing is completed comparing different relations presented in the literature [30-32]: $L_{n}=1.272 r_{n}, r_{\max }=1.252 r_{n}, r_{s}=0.28 r_{n}$ and $L_{s}=1.5 r_{s}$. The shapes of the inlet section are built using Class Shape Transformation (CST) curves, following $[30,33,34]$.

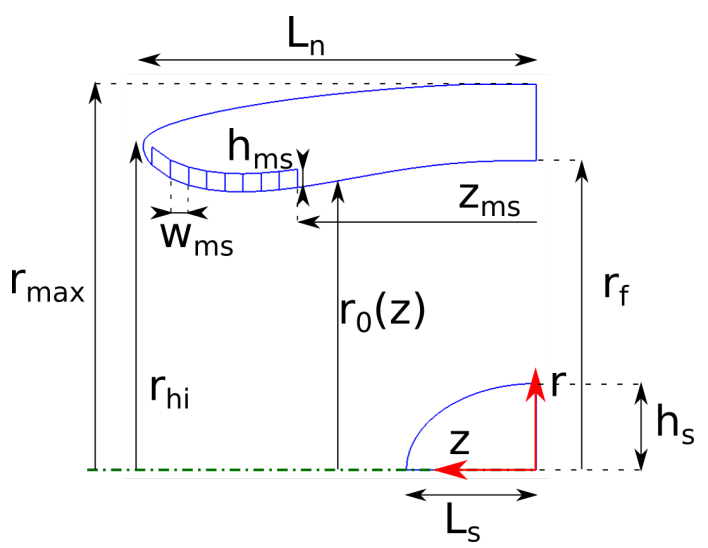

Figure 2. Sketch of the nacelle inlet geometry with the metalining.

The metalining is placed on the internal surface of the inlet, starting from $z_{m s}$ with an overall length of $L_{m s} \approx 0.7 \lambda_{B P F}$ divided into eight cells of equal width $w_{m s}=0.05 \mathrm{~m}$. In the present study, the MS is positioned close to the lip leading edge: the treatment extends in a currently non-lined part of the nacelle as its presence in the nacelle is not intended to be an alternative to the standard absorbing liners. The effect of the positioning of the cells and the total extension of the treatment were not investigated; in future developments, further synergies between the metalinings and the classic acoustic liners may likely arise also considering these two variables.

The main focus is on the reduction of the noise radiated towards the ground from the engine inlet. For this reason, the effect of the MS device is evaluated over an arc of virtual microphones, centred on the middle of the cowling inlet section, with a radius $r_{m i c}=3.5 \mathrm{~m}$ $\left(2.06 r_{n}\right)$. The arc extends from $20^{\circ}$ to $120^{\circ}$ (being $0^{\circ}$ aligned with $z$-direction in Figure 2).

The nacelle is immersed in a quiescent barotropic, inviscid and irrotational fluid, in which the classic wave equation describes the propagation of the acoustic waves, Equation (5). The cells occupy what is from now on called the metafluid domain, where Equation (6) holds the solution for the acoustic pressure, with properties defined by 
Equation (9). The continuity of the acoustic field is ensured at the interface between cells and the outer domain by imposing

$$
p_{m s}=p_{h}, \quad \mathbf{v}_{h} \cdot \mathbf{n}_{h}=-\varrho^{-1} \nabla p \cdot \mathbf{n}_{m s} .
$$

The boundary conditions are completed with perfectly matched layers at the exterior boundaries to truncate the computational domain avoiding non-physical reflections. The internal boundaries of the cells and the walls of the nacelle are considered acoustically rigid, imposing the normal derivative of the acoustic velocity to be null.

The sound source is modelled as a boundary condition on the fan section by considering only one azimuthal mode at the BPF for the maximum rotational speed of the fan $(628 \mathrm{~Hz})$.

The acoustic problem is solved in the frequency domain, the SPLs at the virtual microphones have been evaluated with the commercial FEM solver Comsol ${ }^{\circledR}$, in which Equations (5) and (6), Fourier-transformed, are implemented in their integrated weak formulation and applied to the elements of the discretely meshed domain.

\section{Results}

An optimisation procedure is applied to the metafluid to maximise the noise reduction effect for the selected duct mode propagating in the nacelle. A generic single-objective unconstrained optimisation process can be formalised as follows,

$$
\begin{aligned}
\text { minimize/maximize }[J(\mathbf{x}, \mathbf{y})], & \mathbf{x} \in \mathcal{D} \\
\text { with bounds } x_{n}^{L} \leq x_{n} \leq x_{n}^{U}, & n=1, \ldots, N \\
\text { and } q_{m}=\hat{y}_{m}, & m=1, \ldots, M \text { and } \hat{y}_{m} \in \mathcal{Q}
\end{aligned}
$$

being $J(\mathbf{x}, \mathbf{y})$ the objective function with $\mathbf{x}$ the vector containing the $N$ design variables bounded by $x_{n}^{L}$ and $x_{n}^{U}$ in the design space $\mathcal{D}$, and $\mathbf{y}$ the vector of the $M$ environmental parameters (independent of the designer choice) in the parameter domain $\mathcal{Q}$. For the optimisation of the metafluid design, a merit function is defined as the integral of the acoustic pressure level over the arc of virtual receivers:

$$
J(\mathbf{x}, \mathbf{y})=\int_{\mathcal{L}_{\text {mic }}} 10 \log _{10}\left(\frac{p(\mathbf{x}, \mathbf{y})^{2}}{p_{\text {ref }}^{2}}\right) d l .
$$

The minimisation of the objective functions is achieved through a Particle Swarm Optimization (PSO) algorithm, using a swarm with ten individuals per variable. In the proposed approach, the optimisation variables are the equivalent lengths of the cells $h_{i}$, bounded allowing a range of $\Delta \phi$ of $2 \pi$ for each cell, see Equation (3). The bulk modulus and the inertia tensor of each metafluid cell are then evaluated with Equation (9).

Three different duct modes are used to investigate the effect obtainable from the phase-gradient-based treatment under different incident sound fields. Specifically, results have been obtained for $m=4$ and $m=15$, chosen as representative of low and high-order azimuthal modes. The $m=15$ is associated with only one radial mode with $k=4.408$, as revealed by the numerical modal analysis at the fan section, while, among the five cut-on radial modes for $m=4, k=6.9862$ and $k=11.071$ were considered. Since the duct has a continuously varying cross-section, a preliminary check is done on the selected modes to ensure the propagation of the relative acoustic perturbation.

Figure 3 shows the SPL along the microphone arc comparing the optimised metafluids for each case/mode with the reference solution not including any lining in the duct. A dramatic reduction in the pressure level along the entire arc considered is obtained for the higher-order azimuthal mode $m=15$, while for each of the two $m=4$ modes, the acoustic levels are more effectively lowered starting from $\theta \geq 60^{\circ}$. These reductions cannot be ascribed to absorption by the metasurfaces since the implemented model does 
not consider any dissipative effect. It seems reasonable to explain the observed abatement as the effect of the boundary conditions change in the lined section, such that for $m=15$, the propagation of the considered mode from the inlet section towards the external field is not allowed (evanescent), and for each of the $m=4$ modes, the diffraction from the nacelle lip is strongly reduced along with the side propagation. It is not completely clear to the authors whether this interpretation may be equivalent to a redistribution of the acoustic incident field due to unconventional/extraordinary reflection.

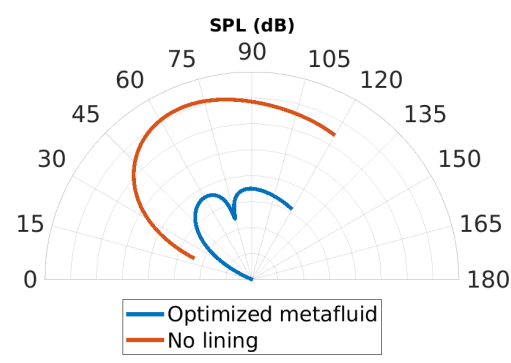

(a)

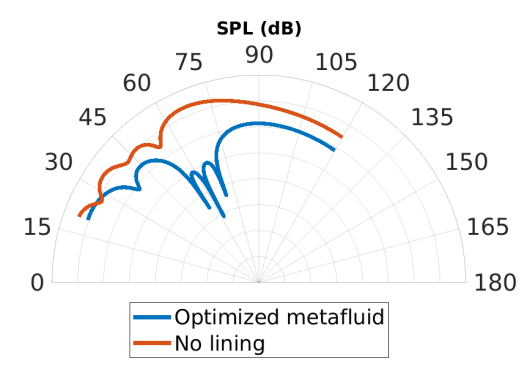

(b)

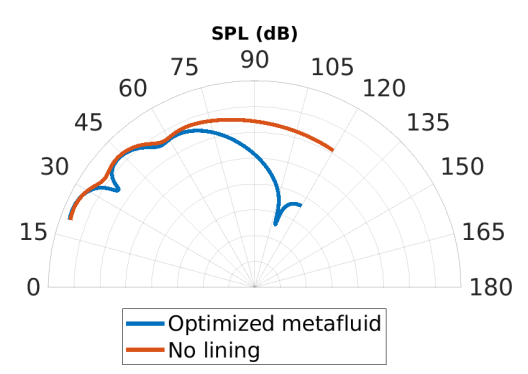

(c)

Figure 3. Sound Pressure Levels over the virtual microphones arc, $M=0.0$; in red, the solution for the optimised metafluid, in blue for the non-lined duct, (a) azimuthal mode $m=15, k=4.408$. (b) Azimuthal mode $m=4, k=6.9862$. (c) Azimuthal mode $m=4, k=11.071$.

The above considerations are confirmed by the field visualization of the acoustic levels around the nacelle in Figure 4. Acoustic energy is not effectively transmitted out of the duct for the $m=15$ case, Figure $4 \mathrm{~b}$, the main emission lobe is highly attenuated for the first $m=4$ mode considered, Figure $4 \mathrm{~d}$, and diffraction from the inlet lip is greatly reduced for both the $m=4$ modes, Figure $4 \mathrm{f}$, causing the reduction in pressure levels for $\theta \geq 60^{\circ}$.

The directivity modification achieved is very different among the three cases considered. It should be noted that the most effective optimised metalining is the one tackling the higher-order azimuthal mode, for which the radiated noise is almost completely eliminated. No general behaviour can be observed in the delay distributions obtained from the optimizations for the three different modes. The optimal delays and their gradients substantially differ from each other as expected, being the only common characteristic a remarkable variability of the delay along the surface.

As already mentioned, the obtained results are preliminary, although encouraging, and aiming to explore the potential of PGMS in a simplified though realistic setting, which includes some of the important features of real applications (such as surface curvatures, varying cross sections, source description). The study is far from the complete design of a liner based on the phase-gradient: metamaterial linings are not a mature technology, and an extensive analysis on their potential benefits and relative exploitation strategy is needed for a technology-readiness level advancement. 


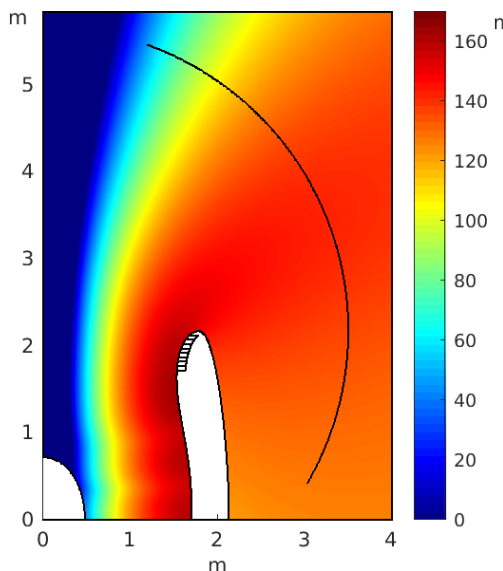

(a)

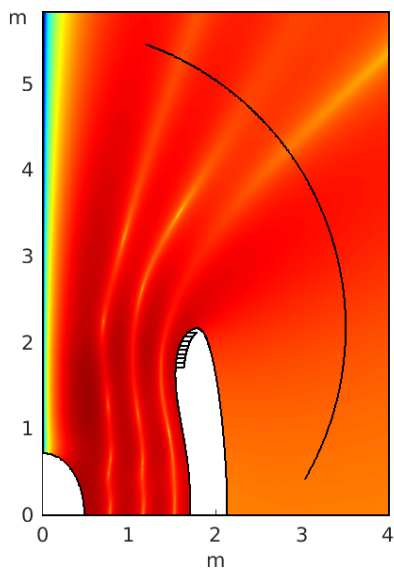

(c)

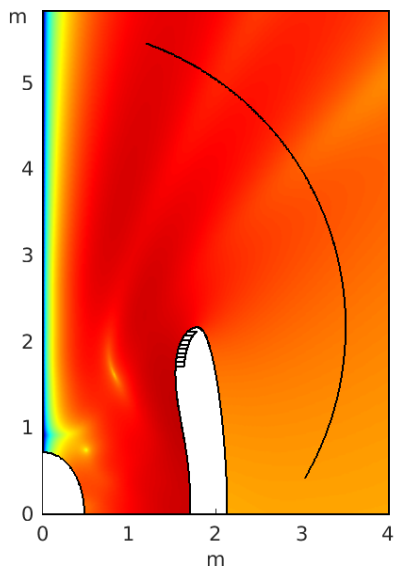

(e)

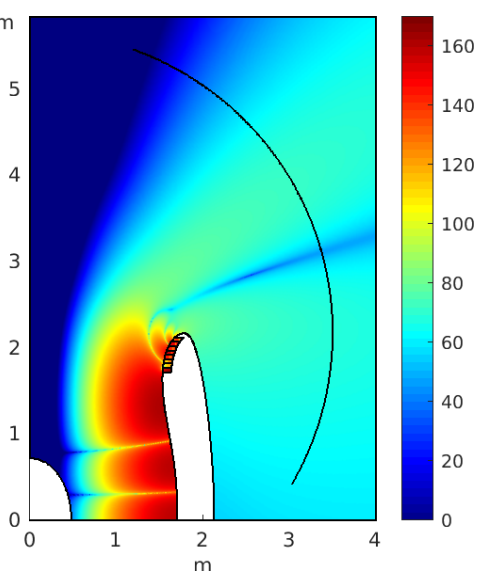

(b)

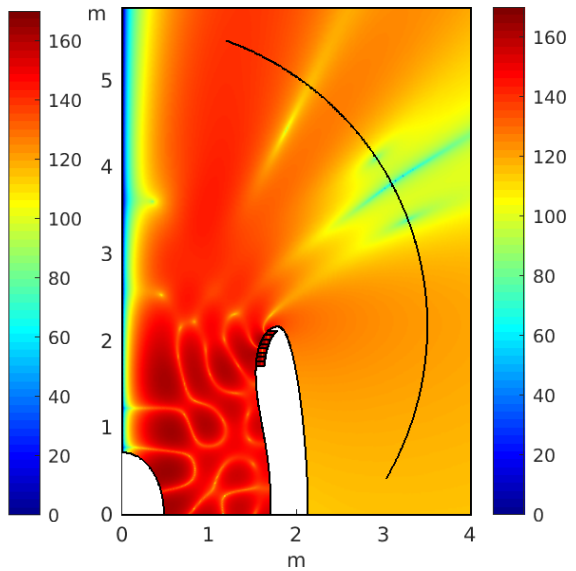

(d)

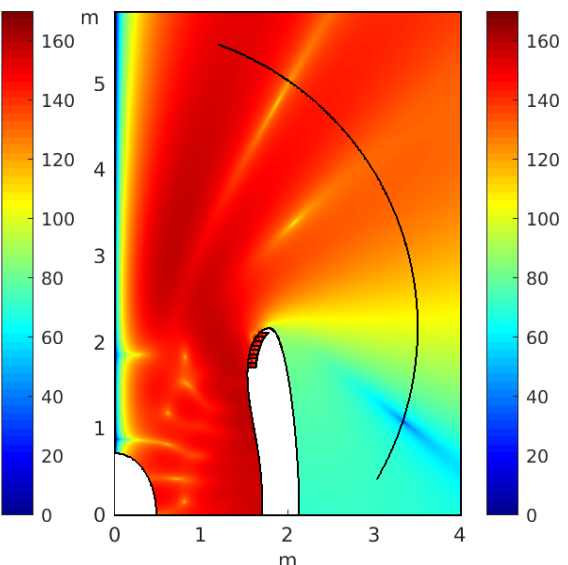

(f)

Figure 4. Sound Pressure Levels around the nacelle inlet, $M=0.0$, the solution for the reference non-lined duct on the left, for the optimised metafluid on the right, $(\mathbf{a}, \mathbf{b})$ azimuthal mode $m=15$, $k=4.408,(\mathbf{c}, \mathbf{d}) m=4, k=6.9862,(\mathbf{e}, \mathbf{f}) m=4, k=11.071$.

\section{Conclusions}

The article presents the extension of phase gradient metasurfaces' analytical and numerical treatment as equivalent metafluids to consider axial symmetric $2.5 \mathrm{D}$ domains. This allows considering geometries with curved surfaces, around the symmetry axis and in the axial direction.

The metafluid model greatly helps perform a simulation-based design optimisation of a PGMS to realise innovative acoustic treatments of realistic nacelle intakes. The acoustic 
source included in the modelling is taken from a modal analysis of the fan section in the inlet duct.

This paper aimed to study the potential benefits of the phase-gradient technology, and is not an attempt to design a complete new liner or nacelle. Simulations appear to confirm the potential of axially non-uniform linings based on metamaterials in the abatement of noise radiated from the nacelles' inlet, already emerged in previous numerical studies. This type of metasurface can steer the reflected field under the quasi-normal incidence of the acoustic field, as evidenced in the literature. However, the effect observed in the present configuration is probably more effectively explained by stating that the lining imposes a boundary condition changing the propagation of the tackled acoustic mode, which in some cases may become evanescent or its diffraction through the inlet section is strongly reduced. The effect is obtained through a metafluid equivalent to rectilinear channels of tailored lengths, which in principle have a purely reactive behaviour, with no energy dissipation.

The analytic model of TA broadens the concepts that can be explored to realise phase gradient metasurfaces and their effects, including all that can tune the occupied domain's effective bulk modulus and density, such as pentamode materials.

No viscothermal acoustic phenomena are modelled in the simulations, and the effects of the aerodynamic flow and boundary layer are not considered. These effects might be considered in future developments using higher fidelity models, with a consequent non-negligible increase in the computational burden.

Optimisations for a single acoustic mode at the BPF have been presented in this work, and each resulting metafluid is not guaranteed to be effective for a different azimuthal/radial mode. Therefore, a multiobjective optimisation might be a viable strategy to extend the window of the metamaterial effectiveness, considering more modes with different axial wavenumber and azimuthal order and/or frequencies of interest. Such an enriched approach comes, however, with an increment of computational cost, linearly increasing with the number of modes/frequencies considered.

Other effects from the metalining may also be explored by using different objective functions, including enhancing the efficacy of standard liners or the virtual scarfing effect, the latter requiring a more expensive complete 3D modelling of the problem.

Author Contributions: Conceptualization, G.P. and L.B.; methodology, G.P. and L.B.; software, G.P. and L.B.; validation, G.P., L.B. and F.C.; formal analysis, G.P.; investigation, G.P.; writing-original draft preparation, G.P., L.B. and F.C.; writing-review and editing, G.P., L.B. and F.C.; visualization, G.P.; supervision, U.I. All authors have read and agreed to the published version of the manuscript.

Funding: This work has been supported by the European Commission through the Project ARTEM (Aircraft noise Reduction Technologies and related Environmental iMpact), Grant Agreement no. 769350.

Institutional Review Board Statement: Not applicable.

Informed Consent Statement: Not applicable.

Conflicts of Interest: The authors declare no conflict of interest.

\section{References}

1. Directorate-General for Mobility and Transport (European Commission); Directorate-General for Research and Innovation (European Commission). Flightpath 2050, Europe's Vision for Aviation; Technical Report; Publications Office of the European Union: Luxemburg, 2011. [CrossRef]

2. Achunche, I.; Astley, J.; Sugimoto, R.; Kempton, A. Prediction of Foward Fan Noise Propagation and Radiation from Intakes. In Proceedings of the 15th AIAA/CEAS Aeroacoustics Conference (30th AIAA Aeroacoustics Conference), Miami, FL, USA, 11-13 May 2009. [CrossRef]

3. Ma, X.; Su, Z. Development of acoustic liner in aero engine: A review. Sci. China Technol. Sci. 2020, 63, 2491-2504. [CrossRef]

4. Gautam, A.; Celik, A.; Azarpeyvand, M. Double Degree of Freedom Helmholtz Resonator Based Acoustic Liners. arXiv 2020, arXiv:2010.05665.

5. Gautam, A.; Celik, A.; Azarpeyvand, M. An Investigation On Neck Extensions For Single and Multi-Degree Of Freedom Acoustic Helmholtz Resonators. arXiv 2020, arXiv:2010.05908. 
6. Flanagan, L.; Heaphy, D.; Kennedy, J.; Leiba, R.; Rice, H. Development of acoustic "meta-liners" providing sub-wavelength absorption. Int. J. Aeroacoust. 2020, 19, 310-323. [CrossRef]

7. Floss, S.; Czwielong, F.; Becker, S.; Kaltenbacher, M. Micro-perforated panels for noise reduction. Elektrotech. Informationstechnik 2021, 138, 171-178. [CrossRef]

8. Palma, G.; Mao, H.; Burghignoli, L.; Göransson, P.; Iemma, U. Acoustic Metamaterials in Aeronautics. Appl. Sci. 2018 , 8, 971. [CrossRef]

9. Iemma, U.; Palma, G. SpaceTime-bending transformations in aeroacoustics. In Proceedings of the 26th International Congress on Sound and Vibration (ICSV 26), Montreal, QC, Canada, 7-11 July 2019.

10. Iemma, U.; Palma, G. Aeroacoustic Design of Metafluid Devices. In Proceedings of the 24th International Congress on Sound and Vibration 2017 (ICSV 24), London, UK, 23-27 July 2017.

11. Huang, X.; Zhong, S.; Stalnov, O. Analysis of scattering from an acoustic cloak in a moving fluid. J. Acoust. Soc. Am. 2014, 135, 2571-2580. [CrossRef]

12. Iemma, U.; Palma, G. On the Use of the Analogue Transformation Acoustics in Aeroacoustics. Math. Probl. Eng. 2017, 2017, 16. [CrossRef]

13. Ryoo, H.; Jeon, W. Effect of compressibility and non-uniformity in flow on the scattering pattern of acoustic cloak. Sci. Rep. 2017, 7, 2125. [CrossRef]

14. He, Y.; Zhong, S.; Huang, X. Extensions to the acoustic scattering analysis for cloaks in non-uniform mean flows. J. Acoust. Soc. Am. 2019, 146, 41-49. [CrossRef]

15. Iemma, U.; Palma, G. Convective correction of metafluid devices based on Taylor transformation. J. Sound Vib. 2019, 443, 238-252. [CrossRef]

16. Iemma, U.; Palma, G. Design of metacontinua in the aeroacoustic spacetime. Sci. Rep. 2020, 10, 18192. [CrossRef] [PubMed]

17. Iemma, U.; Palma, G. Optimization of metasurfaces for the design of noise trapping metadevices. In Proceedings of the 26th International Congress on Sound and Vibration (ICSV 26), Montreal, QC, Canada, 7-11 July 2019.

18. Palma, G.; Burghignoli, L. On the integration of acoustic phase-gradient metasurfaces in aeronautics. Int. J. Aeroacoust. 2020, 19, 294-309. [CrossRef]

19. Palma, G.; Centracchio, F.; Burghignoli, L. Optimized metamaterials for enhanced noise shielding of innovative aircraft configurations. In Proceedings of the 27th International Congress on Sound and Vibration (ICSV 27), Virtual, 11-16 July 2021.

20. Li, Y.; Liang, B.; Gu, Z.m.; Zou, X.Y.; Cheng, J.C. Reflected wavefront manipulation based on ultrathin planar acoustic metasurfaces. Sci. Rep. 2013, 3, 2546. [CrossRef] [PubMed]

21. Li, Y.; Jiang, X.; Li, R.Q.; Liang, B.; Zou, X.Y.; Yin, L.1.; Cheng, J.C. Experimental Realization of Full Control of Reflected Waves with Subwavelength Acoustic Metasurfaces. Phys. Rev. Appl. 2014, 2, 064002. [CrossRef]

22. Zhu, Y.F.; Zou, X.Y.; Li, R.Q.; Jiang, X.; Tu, J.; Liang, B.; Cheng, J.C. Dispersionless manipulation of reflected acoustic wavefront by subwavelength corrugated surface. Sci. Rep. 2015, 5, 10966. [CrossRef]

23. Ding, Y.; Statharas, E.C.; Yao, K.; Hong, M. A broadband acoustic metamaterial with impedance matching layer of gradient index. Appl. Phys. Lett. 2017, 110, 241903. [CrossRef]

24. Dubois, M.; Shi, C.; Wang, Y.; Zhang, X. A thin and conformal metasurface for illusion acoustics of rapidly changing profiles. Appl. Phys. Lett. 2017, 110, 151902. [CrossRef]

25. Wang, X.; Mao, D.; Li, Y. Broadband acoustic skin cloak based on spiral metasurfaces. Sci. Rep. 2017, 7, 11604. [CrossRef]

26. Ge, Y.; Sun, H.x.; Yuan, S.q.; Lai, Y. Broadband unidirectional and omnidirectional bidirectional acoustic insulation through an open window structure with a metasurface of ultrathin hooklike meta-atoms. Appl. Phys. Lett. 2018, 112, 243502. [CrossRef]

27. Norris, A.N. Acoustic metafluids. J. Acoust. Soc. Am. 2009, 125, 839-849. [CrossRef] [PubMed]

28. Kadic, M.; Bückmann, T.; Schittny, R.; Gumbsch, P.; Wegener, M. Pentamode Metamaterials with Independently Tailored Bulk Modulus and Mass Density. Phys. Rev. Appl. 2014, 2, 054007. [CrossRef]

29. Chen, Y.; Liu, X.; Hu, G. Latticed pentamode acoustic cloak. Sci. Rep. 2015, 5, 1-7. [CrossRef] [PubMed]

30. Petrusson, A. Aerodynamic Evaluation of Nacelles for Engines with Ultra High Bypass Ratio. Master's Thesis, Chalmers University of Technology, Gothenburg, Sweden, 2017.

31. Majić, F.; Efraimsson, G.; O’Reilly, C.J. Potential improvement of aerodynamic performance by morphing the nacelle inlet. Aerosp. Sci. Technol. 2016, 54, 122-131. [CrossRef]

32. Sinha, S.; Ojha, S. Rotordynamic Analysis of Asymmetric Turbofan Rotor Due to Fan Blade-out Event with Contact-Impact Rub Loads. In Proceedings of the 53rd AIAA/ASME/ASCE/AHS/ASC Structures, Structural Dynamics and Materials Conference, Honolulu, HI, USA, 23-26 April 2012; pp. 1-31. [CrossRef]

33. Christie, R.; Heidebrecht, A.; MacManus, D. An Automated Approach to Nacelle Parameterization Using Intuitive Class Shape Transformation Curves. J. Eng. Gas Turbines Power 2017, 139, 1-9. [CrossRef]

34. Robinson, M.; MacManus, D.G.; Christie, R.; Sheaf, C.; Grech, N. Nacelle design for ultra-high bypass ratio engines with CFD based optimisation. Aerosp. Sci. Technol. 2021, 113, 106191. [CrossRef] 